\title{
One-Pot Preparation of Fluorinated Mesoporous Silica Nanoparticles for Liquid Marble Formation and Superhydrophobic Surfaces
}

\author{
Adem Yildirim, ${ }^{+, \neq}$Hulya Budunoglu, ${ }^{+, \neq}$Bihter Daglar, ${ }^{+, \neq}$Hakan Deniz, ${ }^{+, \neq}$and Mehmet Bayindir ${ }^{*,+,+, \S}$ \\ ${ }^{\dagger}$ UNAM-National Nanotechnology Research Center, ${ }^{\ddagger}$ Institute of Materials Science and Nanotechnology, and ${ }^{\S}$ Department of Physics, \\ Bilkent University, 06800 Ankara, Turkey
}

Supporting Information

ABSTRACT: One-pot synthesis of fluorinated mesoporous silica nanoparticles (FMSNs) is reported. Uniform mesoporous nanoparticles are prepared by condensation of tetraethyl orthosilicate (TEOS) and fluoroalkyl containing organotriethoxy silane monomers, respectively. The method enables selective deposition of fluorine atoms on the surface of the particles. FMSNs are used to prepare stable liquid marbles with water. An organo-modified silica sol is used with FMSNs to prepare mechanically stable superhydrophobic surfaces (water contact
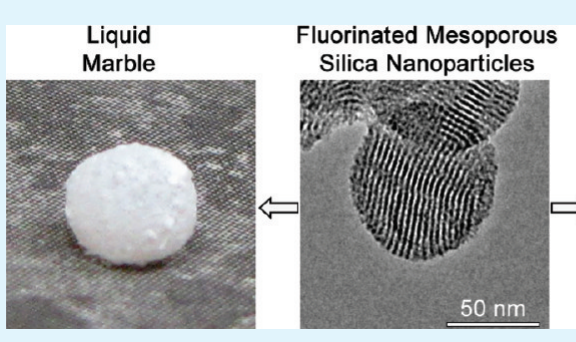

Superhydrophobic

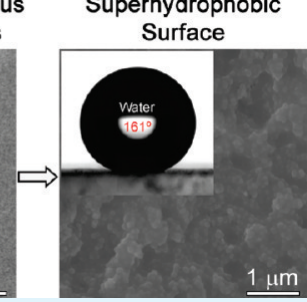

angle of $161^{\circ}$ ). The mechanical stability of the surface is investigated with water dripping and adhesive tape tests. The prepared FMSNs are promising building blocks for robust, large-area, and multifunctional self-cleaning surfaces.

KEYWORDS: superhydrophobic, liquid marble, organo-modified silica, mesoporous, fluoroalkyl-silane, functional surfaces

\begin{abstract}
A ny surface with water contact angle larger than $150^{\circ}$ and Asliding angle lower than $5^{\circ}$ is generally referred as a superhydrophobic surface. ${ }^{1}$ Such extremely water repellent surfaces are attracting a great deal of interest because of their potential applications, including self-cleaning materials, waterproof textiles, and prevention of ice accumulation. ${ }^{2}$ To obtain a superhydrophobic surface, two conditions must be met; micrometerand/or nanometer-sized roughness and low surface energy. ${ }^{3}$ Lithographic methods, ${ }^{4-6}$ chemical etching, ${ }^{7-9}$ or deposition of micrometer- and/or nanometer-sized particles ${ }^{10-14}$ have been usually applied for roughening the surfaces, followed by surface modification with low-surface-energy molecules such as hydrocarbons or fluorinated hydrocarbons. However, such multiplestep fabrication of superhydrophobic surface requires sophisticated equipments and is labor-intensive, which make them impractical for large-area fabrication.

On the other hand, simple single-step coating from solutions where roughness and low-energy surfaces are obtained simultaneously can be more desirable. For example, phase separation in polymers, ${ }^{15}$ electrospinning, ${ }^{16,17}$ sol-gel methods, ${ }^{18,19}$ and hybrid micro- and nano-particles prepared via co-condensation reactions $^{20,21}$ are promising for large-area fabrication of superhydrophobic surfaces. With this insight, we developed a facile single-step method to prepare superhydrophobic surfaces directly applying dispersions of fluorocarbon containing mesoporous silica nanoparticles. Previously, Wang et al. ${ }^{20}$ also reported superhydrophobic surfaces from fluorinated silica particles. They prepared the particles via a modified Stöber method ${ }^{22}$ resulting in a nonporous structure. However, mesoporosity of particles can provide extra functionality to the coatings such as antireflection due to low effective refractive indices. Therefore, we used a surfactant template method ${ }^{23}$ to obtain mesoporous particles.
\end{abstract}

We prepared the fluorinated mesoporous silica nanoparticles (FMSNs) by respective condensation of tetraethyl orthosilicate (TEOS) and perfluorooctyltriethoxysilane (PFOTS) monomers in a one-pot reaction. Surfactant molecules form self-assembled templates, and silica monomers polymerize around these templates. First, TEOS was polymerized forming mesoporous silica nanoparticles (MSNs). Then, PFOTS molecules were added to form a fluorinated layer around these MSNs; thus enabling selective deposition of PFOTS molecules on the surface of MSNs.

As-prepared FMSNs were found to be hydrophobic and formed liquid marbles, which are water droplets enwrapped with hydrophobic particles. ${ }^{24-26}$ To prepare a superhydrophobic surface with FMSNs, we dispersed the particles in an organo-modified silica sol (OSS) (prepared by using methyltrimethoxy silane as precursor) which act as a binder and provide mechanical stability. ${ }^{27}$ The dispersions of FMSNs and OSS were spin coated on glass substrates and heat treated to cure. The coatings have static water contact angles around $160^{\circ}$ and water droplets easily roll off from the surface, both indicating superhydrophobic behavior of the coatings. Furthermore, mechanical stability of the FMSN coating was investigated with water dripping ${ }^{10}$ and adhesive tape ${ }^{28,29}$ tests.

\section{EXPERIMENTAL SECTION}

Tetraetyl orthosilicate (TEOS), Methyltrimethoxysilane (MTMS), isopropanol (IPA), sodium hydroxide and ammonium hydroxide (26\%), were purchased from Merck (Germany), cetyltriammonium

Received: March 22, 2011

Accepted: May 16, 2011

Published: May 16, 2011 
bromide (CTAB), ethanol, and hydrochloric acid (37\%) $(\mathrm{HCl})$ were purchased from Sigma-Aldrich (U.S.A.), 1H,1H,2H,2H-Perfluorooctyltriethoxysilane (PFOTS) was purchased from ABCR (Germany) and methanol, was purchased from Carlo-Erba (Italy) and all chemicals were used as purchased. Fluorinated and non-fluorinated MSNs were prepared by modifying previously reported methods. ${ }^{30-32}$ To prepare FMSNs, we dissolved $0.408 \mathrm{~g}$ of CTAB in $200 \mathrm{~mL}$ of water and added $1.2 \mathrm{~mL}$ of $2 \mathrm{M} \mathrm{NaOH}$. The reaction mixture was heated to $70{ }^{\circ} \mathrm{C}$ under stirring at $750 \mathrm{rpm}$. Then, $2 \mathrm{~mL}$ of TEOS was rapidly added while stirring. After $90 \mathrm{~min}, 0.4 \mathrm{~mL}$ of PFOTS (in $20 \mathrm{~mL}$ of IPA) was slowly added and reaction mixture was further stirred for $90 \mathrm{~min}$. Finally, the particles were filtered and washed with methanol twice and dispersed in ethanol. Nonfluorinated MSNs were prepared without PFOTS addition and reaction mixture was stirred for $2 \mathrm{~h}$ after TEOS addition, whereas other reaction parameters were kept the same as with the FMSN case. To extract CTAB, we dispersed particles in $80 \mathrm{~mL}$ of ethanol and added $1.5 \mathrm{~mL}$ of $\mathrm{HCl}$. The dispersion was heated to $50{ }^{\circ} \mathrm{C}$ and refluxed for $5 \mathrm{~h}$. The extracted particles were collected by centrifugation at $8000 \mathrm{rpm}$ for $20 \mathrm{~min}$, washed with ethanol, and dried over-night in a vacuum oven.

To prepare superhydrophobic surfaces, we prepared OSS by using MTMS as precursor according to our previous report. ${ }^{33}$ Briefly, $2 \mathrm{~mL}$ of MTMS, $2 \mathrm{~mL}$ of ethanol, $0.2 \mathrm{~mL}$ of water, and $5 \mu \mathrm{L}$ of $37 \%$ hydrochloric acid were mixed and stirred at $60^{\circ} \mathrm{C}$. After $90 \mathrm{~min}, 0.4 \mathrm{~mL}$ of $\mathrm{HCl}(0.1$ $\mathrm{M})$ and $0.35 \mathrm{~mL}$ of water were added and the solution was further stirred for $15 \mathrm{~min}$ at room temperature. Finally, the sol was aged at $60^{\circ} \mathrm{C}$ for 15 min and spin-coated onto the glass substrates. FMSN layer was coated on OSS-coated glass substrates by modifying a previously reported method. ${ }^{27}$ First, the $\mathrm{pH}$ of the OSS was adjusted around 9 with ammonia solution (26\%). Then $0.480 \mathrm{~mL}$ of FMSN dispersion in ethanol ( $5 \mathrm{wt} \%$ ) was mixed with the same amount OSS ( $\mathrm{pH}$ 9) and stirred at $60^{\circ} \mathrm{C}$ for 24 $\mathrm{h}$ and spin coated 3 times on previously OSS coated glass substrates. Finally, coatings were cured at $360^{\circ} \mathrm{C}$ for $1.5 \mathrm{~h}$. For control experiments, we prepared a FMSN surface without OSS binder. We dispersed particles $(40 \mathrm{mg})$ in $1 \mathrm{~mL}$ of methanol and spin-coated 3 times on to glass substrates. Finally, coatings were dried at $55^{\circ} \mathrm{C}$ for $1 \mathrm{~h}$.

Transmission electron microscopy (TEM) images were taken using a Tecnai G2 F30 (FEI) microscope. Scanning electron microscopy (SEM) images and Energy Dispersive X-ray Spectroscopy (EDX) spectra were taken with Nova NanoSEM 430 (FEI) microscope at low vacuum. Dynamic Light Scattering (DLS) measurements were performed with Zetasizer Nanoseries (Malvern Instruments). X-ray powder diffraction (XRD) spectra were taken using a Rigaku Miniflex diffractometer. A contact angle meter (OCA 30, Dataphysics) was used to measure the static water contact angle and investigate liquid marble evolution. Water droplets of $0.4 \mu \mathrm{L}$ volume were used and LaplaceYoung fitting was applied on contact angle measurements. The effect of humidity on liquid marble lifetime was investigated at $5 \%$ and $86 \%$ humidity levels in a closed chamber. Silica gel was used to maintain $5 \%$ humidity and saturated $\mathrm{KCl}$ solution for $86 \%$ humidity. ${ }^{26}$ Surface roughness of the films was analyzed using an atomic force microscope (AFM, XE-100E, PSIA) at noncontact mode. Chemical analysis of particles was performed using X-ray photoelectron spectroscopy (XPS, K-Alpha, Thermo Scientific) and thermal gravimetric analysis (TGA, Q500, TA Instruments).

\section{RESULTS AND DISCUSSION}

FMSNs were prepared via respective condensation of TEOS and PFOTS monomers. Following the polymerization of TEOS and formation of MSNs, PFOTS monomer was added in order to obtain fluorinated mesoporous silica shell (Scheme 1). Particle sizes and mesoporous structure of both fluorinated and nonfluorinated mesoporous nanoparticles were investigated by TEM analysis indicating that both MSNs (Figure 1a, b) and FMSNs
Scheme 1. Schematic Representation of Fluorinated Mesoporous Silica Nanoparticle Synthesis, Liquid Marble Formation, and Superhydrophobic Surface Preparation
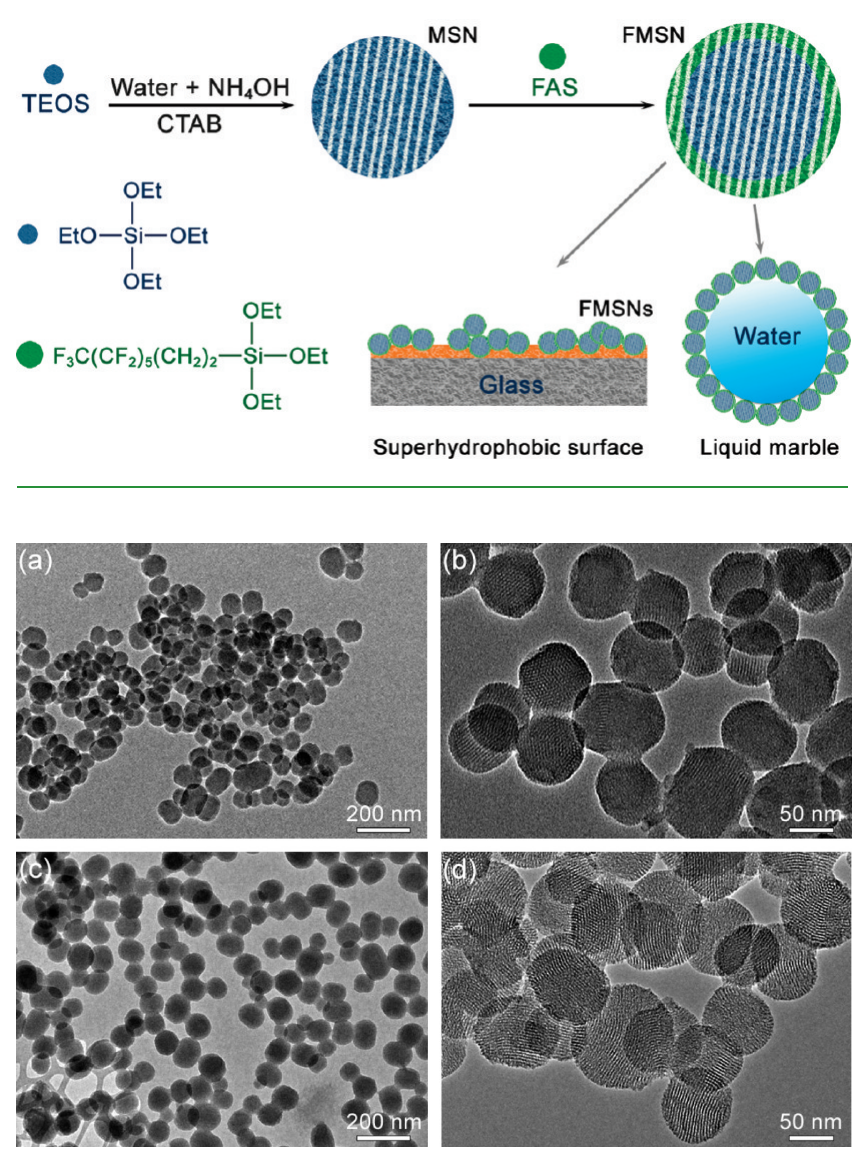

Figure 1. TEM images for ( $a, b)$ MSNs and ( $c, d)$ FMSNs. TEM images demonstrate uniform size distribution and hexagonal mesoporous structure for both particles.

(Figure 1c, d) represent well-ordered hexagonal pore structure. The mesostructure of the particles were also examined by XRD (see the Supporting Information, Figure S1). Both MSNs and FMSNs exhibited characteristic reflection peaks, (100), (110), and (200), of highly ordered hexagonal structures. The d(100) spacing of MSNs and FMSNs were 3.95 and $4.05 \mathrm{~nm}$, respectively. Average particle sizes were calculated from TEM images. FMSNs were 15-20 nm larger than MSNs in size, which confirms the formation of fluorinated shell upon PFOTS addition. Also it must be noted that fluorinated shell may not be formed by condensation of PFOTS molecules alone. Unreacted TEOS molecules in the reaction mixture can contribute to the structure to form a fluorinated mesoporous shell. Particle size distribution was narrow for both MSNs and FMSNs. Smallest and largest particles observed from TEM images are given in Table 1. Sizes of the particles in methanol were also investigated by using DLS method. DLS analysis gives larger particle sizes (Table 1) than TEM analysis because of the particle aggregation in solution. Average particle size of MSNs and FMSNs were found to be 311 and $140 \mathrm{~nm}$, respectively. The particle sizes of MSNs determined with TEM analysis is lower than that of FMSNs, DLS analysis indicates larger particles were formed in methanol for MSNs because surface silanol groups induced particle aggregation. ${ }^{31}$ On the other hand, the uncharged fluoroalykl chains located at the 
Table 1. Synthetic Conditions and Properties of Mesoporous Silica Particles

\begin{tabular}{lcccccc} 
& & \multicolumn{2}{c}{ particle size } & & \multicolumn{2}{c}{ flourine content } \\
\cline { 3 - 4 } & FOS/TEOS & $d_{\text {TEM }}$ & $d_{\text {DLS }}$ & & TGA & XPS \\
& $(\mathrm{v} / \mathrm{v})$ & $(\mathrm{nm})$ & $(\mathrm{nm})$ & & $(\%)$ & $(\%)$ \\
MSN & 0 & $60-100$ & 311 & & \\
FMSN-1 & 0.2 & $70-130$ & 140 & & 10.8 & 11.3 \\
\hline
\end{tabular}

(a)

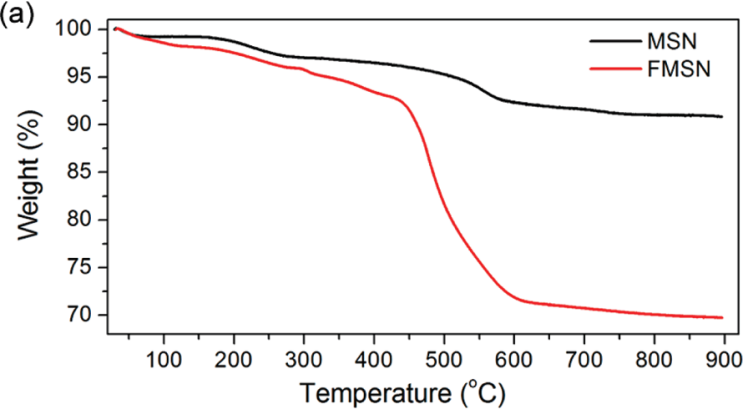

(b)

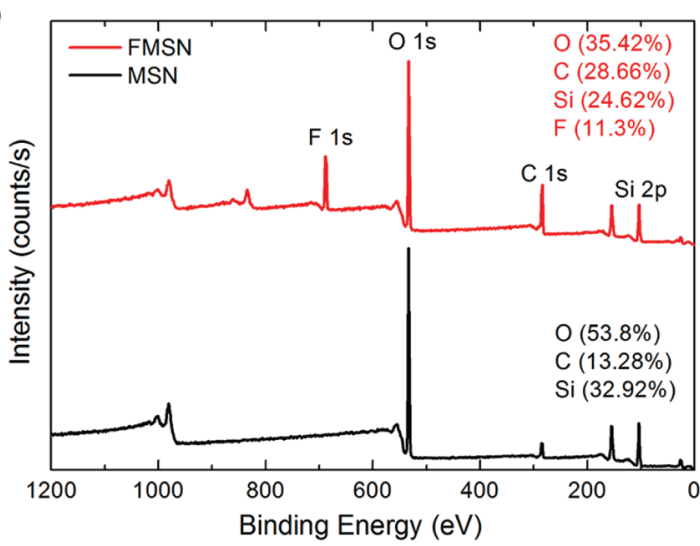

Figure 2. (a) TGA of fluorinated (FMSN) and nonfluorinated (MSN) particles. There is higher weight loss for FMSNs because fluoroalkyl chains decomposed between $450{ }^{\circ} \mathrm{C}$ and $600{ }^{\circ} \mathrm{C}$. (b) XPS spectra of FMSNs and MSNs. Fluorine peak at FMSN spectrum indicates successful fluorination of mesoporous silica particles.

surface decreases the particle aggregation for FMSNs and result in lower particle size in methanol.

The chemical composition of the particles was studied with TGA and XPS. Figure 2a shows TGA analysis of particles. The weight loss for FMSNs was $30.1 \%$ and for MSNs was $9.1 \%$ at $900^{\circ} \mathrm{C}$. The difference of $21 \%$ in weight loss between MSNs and FMSNs can be addressed to the fluoroalkyl chains of the PFOTS monomer. From TGA measurements, the molar fluorine content of FMSNs was calculated to be $10.8 \%$. There is a sharp decrease in the weight of the FMSNs between 450 and $600{ }^{\circ} \mathrm{C}$ where the fluoroalkyl chains of particles were decomposed. Fluoroalkyl chains are stable up to $450{ }^{\circ} \mathrm{C}$ in silica matrix. After that point, they start to decompose and finally at $600{ }^{\circ} \mathrm{C}$ all chains are decomposed. Figure $2 \mathrm{~b}$ shows the XPS spectra of particles. The peak at $687 \mathrm{eV}$ corresponds to $1 \mathrm{~s}$ electron shell of the fluorine. This peak was present only in the spectrum of FMSNs and proves the successful fluorination of particles. The molar ratio of fluorine was $11.3 \%$ based on XPS analysis, which is in good accordance with the results obtained using TGA. Also the small

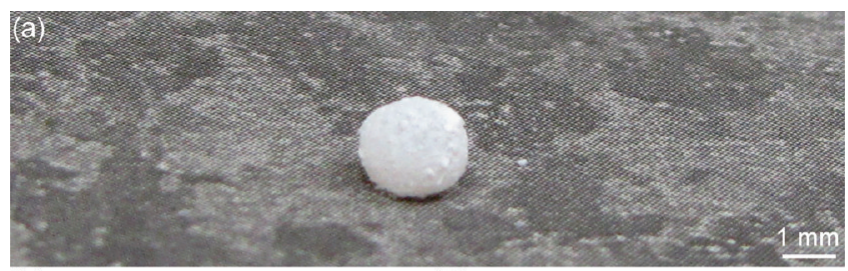

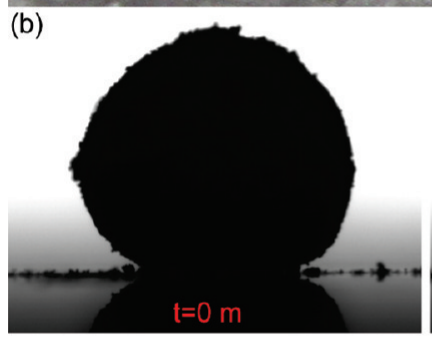

(d)

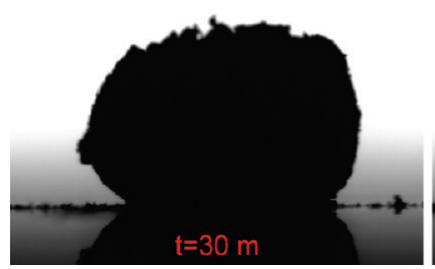

(c)

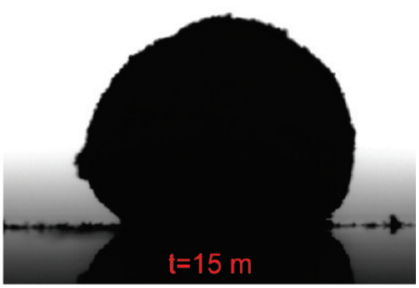

(e)

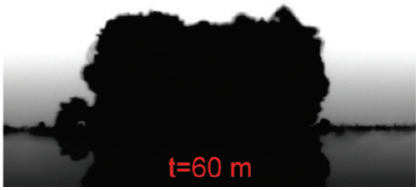

Figure 3. (a) Photograph of a liquid marble of FMSNs prepared with $\sim 12 \mu \mathrm{L}$ of water. (b-e) Evolution of a $12 \mu \mathrm{L}$ water marble with time. The height of the marble is gradually decreased and it shrinks after $60 \mathrm{~min}$.

peak at $832 \mathrm{eV}$ corresponds to the Auger electron of fluorine and again observed only at fluorinated particles. The carbon content of FMSNs is higher than MSNs because of fluoroalkyl chain of PFOTS monomer. Ideally, there must be no carbon in MSNs. However, there is a smaller $\mathrm{C} 1 \mathrm{~s}$ peak at $284 \mathrm{eV}$ that might be due to the residual surfactant molecules and organic contaminants.

FMSN powder formed liquid marbles with water (Figure 3a, see the Supporting Information, Video-1). The fluoroalkyl chains located at the surface of the particles greatly reduce the surface energy and when they are in contact with a water droplet the FMSNs are picked up by the droplet and form a porous shell around it. We formed the liquid marbles using $\sim 2-400 \mu \mathrm{L}$ of water. The smallest and largest marble dimaters were $\sim 1$ and $\sim 13$ $\mathrm{mm}$, respectively. Figure 3a shows a spherical FMSN liquid marble holding $12 \mu \mathrm{L}$ of water. Water evaporation from the FMSN shell were investigated using a contact angle measurement system video camera. Time evolution of a $12 \mu \mathrm{L}$ water marble at room conditions ( $31 \%$ relative humidity, $20^{\circ} \mathrm{C}$ ) is shown in Figure $3 \mathrm{~b}-\mathrm{e}$. Initially, height of the marble started decreasing because of evaporation; after 25 minutes, buckling of the marble occurred; and in $\sim 70$ minutes water evaporated completely from the structure and the marble finally collapsed. The stability of the liquid marbles is similar with previous reports. For example, Fujii et al. ${ }^{34}$ reported a lifetime of $75 \mathrm{~min}$ for $15 \mu \mathrm{L}$ of liquid marbles prepared by using $\sim 600 \mathrm{~nm}$ poly(2-vinylpyridine) particles at room conditions. Also, Erbil and coworkers reported buckling time of $17 \mathrm{~min}$ for $10 \mu \mathrm{L}$ of liquid marbles prepared by using micrometer-sized graphite particles at $40 \%$ humidity ${ }^{26}$ and buckling time of $15 \mathrm{~min}$ and lifetime of $45 \mathrm{~min}$ for $5 \mu \mathrm{L}$ of liquid marbles prepared by using PTFE $(5-6 \mu \mathrm{m})$ particles at $35 \%$ humidity. ${ }^{35} \mathrm{We}$ also examined the effect of humidity on liquid marble lifetime. At lower humidity (5\%) water evaporated faster from the marbles 

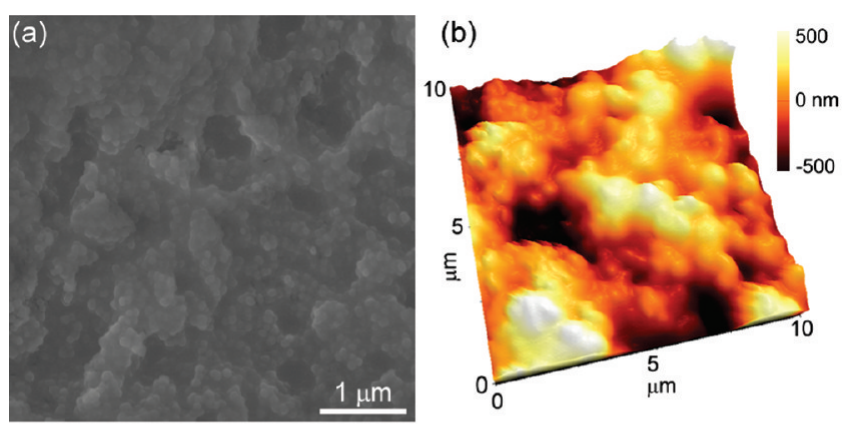

(c)
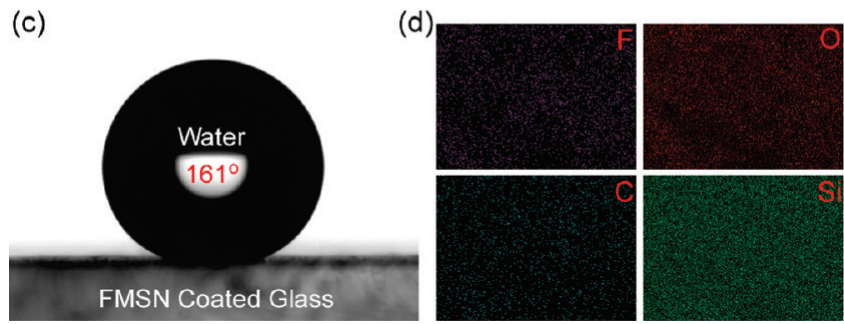

Figure 4. (a) SEM image of superhydrophobic FMSN surface on glass substrate. Particles formed aggregates and result in a very rough surface in dual scale. (b) AFM image showing the surface with average roughness of $246 \mathrm{~nm}$. (c) A $4 \mu \mathrm{L}$ water droplet on the superhydrophobic FMNS surface. (d) EDX maps for F, O, C, and Si, showing homogeneous distribution for all elements.

(45 min) compared to room condition. On the other hand, lifetime of the marbles elongated up to $4 \mathrm{~h}$ at $86 \%$ humidity because of the low evaporation rate.

To produce superhydrophobic surfaces from FMSNs, we dispersed particles in an OSS which was synthesized via polymerization of MTMS monomer in acidic conditions. The OSS act as binder for particles and give the coating mechanical stability. The surface morphology of the FMSN coating was investigated using SEM and AFM analyses. SEM image of the coating (Figure 4a) indicates formation of a rough surface. Individual FMSNs can be clearly seen from the image. The FMSNs form bigger particles by aggregation and result in a two scale roughness which is essential for superhydrophobic surface formation. AFM analysis of the surface (Figure $4 \mathrm{~b}$ ) also demonstrates two scale roughness. Surface roughness of the coating was calculated to be $246 \mathrm{~nm}$ by averaging three $10 \mu \mathrm{m} \times 10 \mu \mathrm{m}$ AFM scans. The combination of low-surface-energy FMSNs and surface roughness of the coatings produce superhydrophobic surface. The static contact angle of the surface was found to be $161.6^{\circ} \pm 4.3^{\circ}$ (Figure 4c) and water droplets did not stick to the surface (See the Supporting Information, Video-2), indicating water droplets were in the Cassie-Baxter state on superhydrophobic FMSN surface. Figure 4d shows the EDX analysis of the superhydrophobic FMSN surface. EDX images reveal that all elements of interest (F, C, Si, O) were distributed homogeneously to the surface.

The mechanical stability of the FMSN superhydrophobic surface was examined with water dripping and adhesive tape tests, and then compared to another FMSN surface prepared in the absence of OSS binder. The control surface was found to be very hydrophobic (water contact angle of $143.1 \pm 1.5^{\circ}$ ). In the water dripping test, both surfaces were treated by falling water droplets $(100 \mu \mathrm{L})$ from $30 \mathrm{~cm}$ at a rate of one drop per second. After exposing the surface to water droplets for $18 \mathrm{~h}$, the surface morphologies was investigated with SEM and contact angle measurement system (see the Supporting Information). We didn't observe any adverse effects on the surface with the binder (see Figure S2b in the Supporting Information), on the other hand, surface without the binder showed cracks (see Figure S2d in the Supporting Information). Water contact angle measurements, however, revealed a decrease for both surfaces after water dripping. FMSN surface without the binder readily deteriorated in $3 \mathrm{~h}$ (contact angle $\sim 115^{\circ}$ ), where the surface with the binder main its superhydrophobicity (contact angle $151^{\circ}$ ). Notably, for the surface prepared without binder, we observed that the water droplet spread slowly, possibly through the formed cracks, and the contact angle decreased gradually reaching a plateau after $30 \mathrm{~s}$. Whereas, the contact angle of the surface prepared by using binder stayed constant (see Figure S3 in the Supporting Information).

Furthermore, we also employed adhesive tape test to demonstrate the mechanical stability of the surface prepared with OSS binder. The surfaces were pressed with adhesive tapes applying approximately $10 \mathrm{kPa}$ and then detached. Surface morphologies were investigated using SEM revealing that the surfaces without binder partially detached whereas surfaces prepared with binder remains intact (see the Supporting Information, Figure S4). These observations prove that the OSS improves mechanical stability of the FMSN surface by binding the particles.

\section{CONCLUSION}

We described a facile one-pot method for production of fluorinated mesoporous silica nanoparticles. The prepared particles formed stable liquid marbles with water indicating successful fluorination of the particles. Fluorination of mesoporous silica particles was also confirmed with XPS and TGA analyses. The FMSNs were used to prepare a mechanically stable superhydrophobic surface on glass substrate. For this purpose fluorinated particles were mixed with an organo-modified silica sol to give mechanical stability. The mechanical stability of coatings was demonstrated with water dripping and adhesive tape tests. The FMSN coating maintained its superhydrophobicity and integrity even after $3 \mathrm{~h}$ of excessive water dripping and adhesive tape treatment. Furthermore, the FMSNs can be used in catalysis, controlled release, separation, and sensor applications where mesoporosity, high surface area, and hydrophobicity are essential.

\section{ASSOCIATED CONTENT}

Supporting Information. (1) A PDF containing XRD data of particles and additional SEM images and water contact angle data of the surfaces. (2) A video showing liquid marble formation with water. (3) A video showing non-sticky superhydrophobic FMSN surface. This material is available free of charge via the Internet at http://pubs.acs.org.

\section{AUTHOR INFORMATION}

\section{Corresponding Author}

*Tel: +90 312 2903500. Fax: +90 312266 4365. E-mail: bayindir@nano.org.tr.

\section{ACKNOWLEDGMENT}

We thank Mecit Yaman for fruitful discussions and Mustafa Güler for TEM images. This work is supported by TÜB ITAK under the Project 106G090. M.B. acknowledges support from the Turkish Academy of Sciences Distinguished Young Scientist Award (TUBA GEBIP). 


\section{REFERENCES}

(1) Li, X. M.; Reinhoudt, D.; Crego-Calama, M. Chem. Soc. Rev. 2007, 36, 1350.

(2) Gould, P. Mater. Today 2003, 6, 44.

(3) Sun, T. L.; Feng, L.; Gao, X. F.; Jiang, L. Acc. Chem. Res. 2005, 38,644 .

(4) Oner, D.; McCarthy, T. J. Langmuir 2000, 16, 7777.

(5) McHale, G.; Aqil, S.; Shirtcliffe, N. J.; Newton, M. I.; Erbil, H. Y. Langmuir 2005, 21, 11053.

(6) Shieh, J.; Hou, F. J.; Chen, Y. C.; Chen, H. M.; Yang, S. P.;

Cheng, C. C.; Chen, H. L. Adv. Mater. 2010, 22, 597.

(7) Dorrer, C.; Rühe, J. Adv. Mater. 2008, 20, 159.

(8) Liu, X.; He, J. Langmuir 2009, 25, 11822.

(9) Shi, F.; Chen, X.; Wang, L.; Niu, J.; Yu, J.; Wang, Z.; Zhang, X. Chem. Mater. 2005, 17, 6177.

(10) Li, Y.; Liu, F.; Sun, J. Chem. Commun. 2009, 2730.

(11) Li, X.; Du, X.; He, J. Langmuir 2010, 26, 13528.

(12) Zhai, L.; Cebeci, F. C.; Cohen, R. E.; Rubner, M. F. Nano Lett. 2004, 4, 1349.

(13) Xu, Q. F.; Wang, J. N.; Sanderson, K. D. ACS Nano 2010, 4, 2201.

(14) Cao, L.; Gao, D. Faraday Discuss. 2010, 146, 57.

(15) Erbil, H. Y.; Demirel, A. L.; Avci, Y.; Mert, O. Science 2003, 299, 1377.

(16) Acatay, K.; Simsek, E.; Ow-Yang, C.; Menceloglu, Y. Z. Angew. Chem., Int. Ed. 2004, 43, 5210.

(17) Ma, M.; Hill, R. M.; Lowery, J. L.; Fridrikh, S. V.; Rutledge, G. C. Langmuir 2005, 21, 5549.

(18) Barkhudarov, P. M.; Shah, P. B.; Watkins, E. B.; Doshi, D. A.; Brinker, C. J.; Majewski J. Corros. Sci. 2008, 50, 897.

(19) Budunoglu, H.; Yildirim, A.; Guler, M. O.; Bayindir, M. ACS Appl. Mater. Interfaces 2011, 3, 539.

(20) Wang, H. X.; Fang, J.; Chang, T.; Ding, J.; Qu, L. T.; Dai, L. M.; Wang, X. G.; Lin, T. Chem. Commun. 2008, 877.

(21) Bonilla, A. M.; Bousquet, A.; Ibarboure, E.; Papon, E.; Labrugere, C.; Rodriguez-Hernandez, J. Langmuir 2010, 26, 16775.

(22) Stöber, W.; Fink, A.; Bohn, E. J. Colloid Interface Sci. 1968, 26,62 .

(23) Fowler, C. E.; Khushalani, D.; Lebeau, B.; Mann, S. Adv. Mater. 2001, 13, 649 .

(24) Aussillous, P.; Quere, D. Nature 2001, 411, 924.

(25) Bormashenko, E.; Balter, R.; Aurbach, D. Appl. Phys. Lett. 2010, 97, 091908.

(26) Dandan, M.; H. Erbil, H. Y. Langmuir 2009, 25, 8362.

(27) Manca, M.; Cannavale, A.; De Marco, L.; Arico, A. S.; Cingolani, R; Gigli, G. Langmuir 2009, 25, 6357.

(28) Ling, X. Y.; Phang, I. Y.; Vansco, G. J.; Huskens, J.; Reinhoudt, D. N. Langmuir 2009, 25, 3260.

(29) Deng, X.; Mammen, L.; Zhao, Y.; Lellig, P.; Müllen, K.; Li, C.; Butt, H. -J.; Vollmer, D. Adv. Mater. 2011 published online.

(30) Cai, Q.; Luo, Z. S.; Pang, W. Q.; Fan, Y. W.; Chen, X. H.; Cui, F. Z. Chem. Mater. 2001, 13, 258.

(31) Liong, M.; Lu, J.; Kovochich, M.; Xia, T.; Ruehm, S. G.; Nel, A. E.; Tamanoi, F.; Zink, J. I. ACS Nano 2008, 2, 889.

(32) Kim, J.; Kim, H. S.; Lee, N.; Kim, T.; Kim, H.; Yu, T.; Song, I. C.; Moon, W. K.; Hyeon, T. Angew. Chem., Int. Ed. 2008, 47, 8438.

(33) Yildirim, A.; Budunoglu, H.; Deniz, H.; Guler, M. O.; Bayindir, M. ACS Appl. Mater. Interfaces 2010, 2, 2892.

(34) Fujii, S.; Kameyama, S.; Armes, S. P.; Dupin, D.; Suzaki, M.; Nakamura, Y. Soft Matter 2010, 6, 635.

(35) Tosun, A.; Erbil, H. Y. Appl. Surf. Sci. 2009, 256, 1278. 